\title{
Detecting $K R A S$ mutations in peripheral blood of colorectal cancer patients by peptide nucleic acid clamp PCR
}

\author{
SHOZO MIYANO $^{1 *}$, KISABURO HANAZAWA ${ }^{1 *}$, TOSHIAKI KITABATAKE $^{2}$, \\ MINORU FUJISAWA $^{2}$ and KUNIAKI KOJIMA ${ }^{2}$ \\ Departments of ${ }^{1}$ Oncology and ${ }^{2}$ General Surgery, Juntendo University Nerima Hospital, Tokyo 177-8521, Japan
}

Received June 1, 2012; Accepted August 28, 2012

DOI: $10.3892 /$ etm.2012.694

\begin{abstract}
We investigated the effectiveness of peptide nucleic acid (PNA) clamp PCR for detecting KRAS mutations in peripheral blood samples of colorectal cancer (CRC) patients. We compared KRAS point mutations between tumour tissue and blood samples. Forty-two patients were included in this study. We observed KRAS mutations in formalin-fixed, paraffin-embedded tissues by PCR direct sequencing and in blood samples by PNA clamp PCR. KRAS point mutations were detected in primary tumour tissue samples of 13 patients $(31.0 \%)$ and in peripheral blood samples of 10 patients (23.8\%). KRAS point mutations were detected in both samples for 8 patients (19.0\%). The sensitivity, specificity and accuracy for detecting KRAS mutations in peripheral blood and tumour tissue samples were $61.5,93.1$ and $83.3 \%$, respectively. The positive and negative predictive values were 80.0 and $84.4 \%$, respectively. Five patients with mutant KRAS in their plasma preoperatively, did not exhibit $K R A S$ mutations postoperatively. Our method detected KRAS point mutations in peripheral blood samples of CRC patients, which contained extremely small amounts of mutant cells. This method is helpful for identifying metastatic CRC patients in whom metastases will respond to EGFR-targeted monoclonal antibody therapy.
\end{abstract}

\section{Introduction}

Colorectal cancer (CRC) is the third most common cancer in the world (1). Extensive research has improved the survival of CRC patients, but its disease-specific mortality remains to be approximately $33 \%$ in the developed world (2). Recently, cetuximab and panitumumab, two monoclonal antibodies targeting the epidermal growth factor receptor (EGFR), have

Correspondence to: Dr Kisaburo Hanazawa, Department of Oncology, Juntendo University Nerima Hospital, Takanodai 3-1-10, Nerima-ku, Tokyo 177-8521, Japan

E-mail: hanazawa@juntendo-nerima.jp

*Contributed equally

Key words: KRAS mutation, colorectal cancer, peptide nucleic acid, plasma proven to be effective in combination with chemotherapy for treating CRC (3). Data from oxaliplatin and cetuximab in firstline treatment of metastatic colorectal cancer and cetuximab combined with irinotecan in first-line therapy for metastatic colorectal cancer trials have indicated that the addition of cetuximab during first-line treatment with infusional fluorouracil, leucovolin and oxaliplatin or fluorouracil, leucovolin and irinotecan was not beneficial in KRAS-mutated tumours, although these patients benefited from chemotherapy alone $(4,5)$. KRAS point mutations are predictive markers for effective treatment with EGFR inhibitors (6).

Cetuximab and panitumumab have been approved by the US Food and Drug Administration. Both of these molecules bind to the extracellular domain of EGFR, leading to inhibition of its downstream signalling (7). EGFR is a transmembrane receptor tyrosine kinase belonging to the ErbB (also known as HER) family that is activated in many tumours. EGFR overexpression by cancer cells results in ligand-independent dimerization and activation (8). KRAS encodes a small $\mathrm{G}$ protein that links ligand-dependent receptor activation to intracellular pathways of the EGFR signalling cascade. Mutation at key sites within the gene, commonly at codons 12 and 13, causes constitutive activation of $K R A S$-associated signalling (5).

Many studies have examined new predictive factors in tumour tissue and blood samples. However, none of these markers have been introduced into clinical practice for CRC treatment, except for KRAS mutations as predictors of non-response to anti-EGFR treatment (9). In this study, we investigated the effectiveness of KRAS mutations in the blood samples of CRC patients to predict response to anti-EGFR therapy. We compared KRAS point mutations in tumour tissues and peripheral blood samples. Peptide nucleic acid (PNA) clamp PCR was used to detect mutations in codons 12 and 13 of the KRAS gene. PNA is a synthetic DNA analogue in which the ribose-phosphate backbone of the DNA is replaced by peptide bond-linked N-(2-aminoethyl)-glycine units. PNA clamp real-time PCR provides a time-sparing and sensitive method for the detection of KRAS point mutations using blood samples of CRC patients (10).

\section{Patients and methods}

Forty-two eligible patients were enrolled in this study between September, 2009 and March, 2010. None of the patients had 
Table I. Primers and PNA clamp probe sequences.

\begin{tabular}{ll} 
Name & \multicolumn{1}{c}{ Sequences } \\
\hline$K R A S-\mathrm{F}$ & 5'-TGTGTGACATGTTCTAATATAGTCACATTT-3 \\
$K R A S-\mathrm{R}$ & 5'-ATCGTCAAGGCACTCTTGCCTAC-3' \\
PNA clamp probe & 5'-TACGCCACCAGCTCC-3'
\end{tabular}

PNA, peptide nucleic acid; F, forward; R, reverse.

Table II. Patient characteristics.

\begin{tabular}{lc}
\hline Characteristics & No. of patients $(\%)$ \\
\hline Patients & 42 \\
Median age (range), in years & $67.5(33-84)$ \\
Gender & \\
Male & $30(71.4)$ \\
Female & $12(28.6)$ \\
Primary tumour site & \\
Colon & $29(69.0)$ \\
Rectum & $13(31.0)$
\end{tabular}

Histopathological subtype

Adenocarcinoma

Adenocarcinoma with a

mucinous component

Mucinous adenocarcinoma

Differentiation

Well

$25(59.5)$

Moderately

Poorly

Depth of tumour invasion (T stage)

$\mathrm{T} 1$

T2

$\mathrm{T} 3$

T4

Unknown

Lymph node metastases ( $\mathrm{N}$ stage)

$\begin{array}{lr}\text { N0 } & 22(52.4) \\ \text { N1 } & 10(23.8) \\ \text { N2 } & 8(19.0) \\ \text { Unknown } & 2(4.8) \\ \text { Stage (TNM) } & \\ 0 & 1(2.4) \\ \text { I } & 10(23.8) \\ \text { IIA } & 10(23.8) \\ \text { IIB } & 3(7.1) \\ \text { IIIA } & 0 \\ \text { IIIB } & 6(14.3) \\ \text { IIIC } & 7(16.7) \\ \text { IV } & 5(11.9)\end{array}$

received previous therapy for CRC. A 5-ml peripheral blood sample was obtained from each CRC patient before tumour resection or endoscopic biopsy. We detected KRAS mutations in formalin-fixed, paraffin-embedded (FFPE) tissue samples by PCR direct sequencing and in blood samples by PNA clamp PCR. This study was approved by the Ethics Committee of Juntendo University. Written informed consent for conducting tumour tissue and blood research was obtained from all patients.

PCR direct sequencing of FFPE samples. DNA was purified from FFPE tissue samples of CRC patients using the QIAamp DNA FFPE tissue kit (Qiagen, Venlo, The Netherlands) as described by the manufacturer's instructions. PCR amplification of exon 2 of the KRAS gene was performed in a total volume of $25 \mu \mathrm{l}$ containing $1 \mathrm{X}$ PCR buffer, $200 \mathrm{nM}$ dNTPs, $1.5 \mathrm{mM} \mathrm{MgCl}_{2}, 100 \mathrm{nM}$ forward and reverse primers, 0.5 units of Platinum TaqDNA polymerase (Invitrogen, Carlsbad, CA, USA) and $25 \mathrm{ng}$ of template DNA. Thermal cycling conditions were as follows: initial denaturation at $94^{\circ} \mathrm{C}$ for $2 \mathrm{~min}$, 40 cycles at $94^{\circ} \mathrm{C}$ for $30 \mathrm{sec}$ and $60^{\circ} \mathrm{C}$ for $1 \mathrm{~min}$ and final elongation at $72^{\circ} \mathrm{C}$ for $10 \mathrm{~min}$. PCR products were purified by the Illustra ${ }^{\mathrm{TM}}$ GFX PCR DNA and Gel Band Purification kit (GE Healthcare, Little Chalfont, UK). DNA sequencing of PCR products was performed on a Genetic Analyzer 3130xl (Applied Biosystems, Foster City, CA, USA) using the BigDye ${ }^{\circledR}$ Terminator v1.1 Sequencing kit (Applied Biosystems) as described by the manufacturer's instructions.

PNA clamp PCR of plasma samples (10). DNA was purified from $3 \mathrm{ml}$ of plasma samples of CRC patients using the QIAamp Circulating Nucleic Acid kit (Qiagen) as described by the manufacturer's instructions. PNA clamp PCR was performed in a total volume of $25 \mu \mathrm{l}$ containing $1 \mathrm{X}$ Phusion HF buffer, $200 \mathrm{nM}$ dNTPs, $60 \mathrm{nM}$ forward and reverse primers, 500 nM PNA probe (Table I), 31.25-fold diluted SYBR-Green (1:2,000 in DMSO), 0.4 units of Phusion HF DNA polymerase (Finnzymes, Espoo, Finland and $2.5 \mu \mathrm{l}$ of plasma DNA. Thermal cycling conditions were as follows: initial denaturation at $98^{\circ} \mathrm{C}$ for $30 \mathrm{sec}, 40$ cycles at $98^{\circ} \mathrm{C}$ for $10 \mathrm{sec} ; 76^{\circ} \mathrm{C}$ for $10 \mathrm{sec} ; 62^{\circ} \mathrm{C}$ for $20 \mathrm{sec}$ and $72^{\circ} \mathrm{C}$ for $10 \mathrm{sec}$ followed by a final cycle at $95^{\circ} \mathrm{C}$ for $15 \mathrm{sec}$ and $60^{\circ} \mathrm{C}$ for $15 \mathrm{sec}$ and dissociation at $95^{\circ} \mathrm{C}$ for $15 \mathrm{sec}$. The reaction was performed using the Sequence Detection System Prism 7900HT System (Applied Biosystems). Each sample was analysed with and without PNA. PCR products were purified by ExoSAP-IT ${ }^{\circledR}$ (USB, Cleveland, OH, USA). DNA sequencing of PCR products was performed on a Genetic Analyzer 3130xl using the BigDye 
Table III. Detection of KRAS mutations in tumour tissue samples and in peripheral blood samples.

\begin{tabular}{|c|c|c|c|}
\hline & \multicolumn{3}{|c|}{$K R A S$ mutation sites } \\
\hline & $\begin{array}{c}\text { KRAS mutations } \\
\mathrm{n}(\%)\end{array}$ & $\begin{array}{l}\text { Codon } 12 \\
\mathrm{n}(\%)\end{array}$ & $\begin{array}{l}\text { Codon } 13 \\
\mathrm{n}(\%)\end{array}$ \\
\hline$K R A S$ in tumor tissue samples & $13(31.0)$ & $10(23.8)$ & $3(7.1)$ \\
\hline$K R A S$ in peripheral blood samples & $10(23.8)$ & $6(14.3)$ & $4(9.5)$ \\
\hline Blood and tissue samples & $8(19.0)$ & $6(14.3)$ & $2(4.8)$ \\
\hline
\end{tabular}

Table IV. Correlation between PCR sequencing and PNA clamp PCR in tumour tissue and peripheral blood.

\begin{tabular}{lccr}
\hline & \multicolumn{3}{c}{ KRAS in tumor tissue } \\
\cline { 2 - 3 } & Positive & Negative & \\
\hline$K R A S$ in peripheral blood & & 2 & $<0.0005$ \\
Positive & 8 & 27 & \\
Negative & 5 & & \\
\hline
\end{tabular}

Sensitivity $61.5 \%$, specificity $93.1 \%$, accuracy $83.3 \%$, positive predictive value $80.0 \%$ and negative predictive value $84.4 \%$.

Table V. Eight patients with KRAS mutations in both the primary tumour tissue and peripheral blood samples.

\begin{tabular}{lllllll}
\hline Patient & Gender & Age & Mutation site & Differentiation & T & N \\
\hline 1 & Female & 71 & Gly13Asp & Moderately & 3 & 0 \\
2 & Female & 81 & Gly12Val & Moderately & 3 & IIA \\
3 & Male & 65 & Gly12Asp & Well & 4 & 0 \\
4 & Male & 72 & Gly12Ser & Moderately & 4 & IIA \\
5 & Female & 51 & Gly13Asp & Moderately & 3 & 2 \\
6 & Female & 78 & Gly12Val & Moderately & 3 & IIIB \\
7 & Female & 72 & Gly12Ser & Well & 3 & IIIC \\
8 & Male & 74 & Gly12Cys & Moderately & 3 & IIV \\
\hline
\end{tabular}

Terminator v1.1 Sequencing kit as described by the manufacturer's instructions.

\section{Results}

We studied 42 patients with CRC, including 30 men and 12 women, with a median age of 67.5 years (range, 33-84 years) from September, 2009 to March, 2010. Patient characteristics are summarized in Table II. Twenty-nine tumours (69.0\%) were present in the colon and $13(31.0 \%)$ were present in the rectum. The primary tumours of 40 patients were resected surgically, and tumour samples of the remaining 2 patients were obtained by endoscopic biopsy. Histologically, 25 (59.5\%) tumours were well-differentiated carcinomas, $16(38.1 \%)$ were moderately differentiated carcinomas and $1(2.4 \%)$ was a poorly differentiated carcinoma.

$K R A S$ point mutations were detected in primary tumour tissue samples of 13 patients $(31.0 \%)$ and in peripheral blood samples of $10(23.8 \%)$. KRAS point mutations were found in both samples for 8 patients (19.0\%). Primary tumour tissue samples of 10 patients showed KRAS point mutations in codon 12 and those of 3 patients showed mutations in codon 13. Plasma samples of 6 patients showed KRAS point mutations in codon 12 and those of 4 patients showed mutations in codon 13 (Table III).

The sensitivity, specificity and accuracy of the assay for detecting KRAS mutations in peripheral blood and tumour tissue samples were $61.5,93.1$ and $83.3 \%$, respectively. The positive and negative predictive values were 80.0 and $84.4 \%$, respectively (Table IV). Two patients showed KRAS point mutations only in their peripheral blood samples. Thirteen patients showed KRAS point mutations in primary tumour tissue samples, and among them 4 had a Gly12Val mutation, 3 had a Gly13Asp mutation, 3 had a Gly12Asp mutation, 2 had a Gly12Ser mutation and 1 had a Gly12Cys mutation. Ten patients showed KRAS point mutations in serum samples, and among them 4 had a Gly13Asp mutation, 2 had a Gly12Ser mutation, 2 had a Gly12Val mutation, 1 had a Gly12Asp mutation and 1 
Table VI. Two patients with KRAS point mutations in the blood samples only.

\begin{tabular}{ccccccccr}
\hline Patient & Gender & Age & Mutation site & Differentiation & T & N & M & Stage \\
\hline 9 & Male & 80 & Gly13Asp & Moderately & 3 & 0 & 0 & IIA \\
10 & Male & 63 & Gly13Asp & Moderately & 3 & 0 & 0 & IIA \\
\hline
\end{tabular}

Table VII. KRAS analysis of plasma preoperatively and 6 months postoperatively.

\begin{tabular}{llccc}
\hline & & & \multicolumn{2}{c}{ Plasma KRAS mutation site } \\
\cline { 4 - 5 } Patient & Gender & Age & Preoperatively & Postoperatively \\
\hline 1 & Female & 71 & Gly13Asp & Wild-type \\
2 & Female & 81 & Gly12Val & Wild-type \\
4 & Male & 72 & Gly12Ser & Wild-type \\
5 & Female & 51 & Gly13Asp & Wild-type \\
6 & Female & 78 & Gly12Val & Wild-type \\
\hline
\end{tabular}

had a Gly12Cys mutation. Five patients showed KRAS point mutations only in their primary tumour tissue samples. Eight patients showed KRAS point mutations in both their primary tumour and blood samples, and we found a concordance of KRAS mutation status between blood and primary tumour tissue samples in all 8 patients (Table V). Two patients showed KRAS point mutations only in the blood samples, and both patients had a Gly13Asp mutation (Table VI). After 6 months, we investigated peripheral blood samples from 5 patients who had mutant KRAS in their plasma preoperatively. None of the patients exhibited KRAS mutants postoperatively (Table VII).

\section{Discussion}

The Catalogue of Somatic Mutations in Cancer database reported KRAS mutations in a total of 8402 samples and no mutations in 29,328 samples (11). Point mutations were most commonly observed in codons 12 and 13 of the KRAS gene and less commonly in codon 61 (12). KRAS mutations have been found in $65-100 \%$ of pancreatic carcinomas, $36 \%$ of CRCs and $20 \%$ of non-small cell lung cancers (13). We detected KRAS mutations in $31.0 \%$ of the tumour tissue samples in our cases. Thus, our results are consistent with those obtained by other studies.

KRAS mutation analysis of primary tumour tissue samples is performed since metastatic tissue is often not available. KRAS genotyping in metastatic CRC patients may be prevented due to the difficulty in obtaining tumour samples from certain patients. Considering the genetic heterogeneity of CRCs, the absence of detectable KRAS mutations in primary tumour tissue samples does not exclude the presence of KRAS mutations in metastatic tissues. Whether metastatic CRC patients carry the same KRAS mutation as the primary tumour is controversial. Furthermore, whether KRAS mutations are stable or whether they undergo clonal evolution during tumour progression remains a matter of debate. Few studies have reported the presence of mutations in metastatic tissues derived from CRCs or the absence of mutations present in the primary tumour tissues in the distant recurrence (14). Klein suggested that tumour cells detach from the primary lesion before acquisition of a malignant phenotype to develop new mutations and exhibit metastatic growth at a distant site in an early dissemination model (15). The variation in mutations between primary tumours and metastases may occur, and consequently, mutations in the primary tumour may not be sufficient to predict the response of metastases to EGFR-targeted monoclonal antibodies. Some studies found a discordance of KRAS mutations in primary tumours and metastatic sites, with an overall discordance observed in $4-32 \%$ of patients. It is still debatable whether the assessment of KRAS mutations in the available primary tumour accurately indicates $K R A S$ mutations in the corresponding metastasis (16).

Recently, Knijn et al (16) reported a concordant KRAS mutation status in $96.4 \%$ of 305 paired samples of colorectal tumours and liver metastases. A discordant KRAS status between primary tumour and metastatic tissues was observed in a small number of patients (3.6\%). KRAS mutations are believed to emerge early during CRC progression and are associated with the growth of small adenomas to a clinically significant size (17). Therefore, KRAS mutations are predicted to be identical in primary tumour and metastatic tissues (15).

There is evidence that primary cancer begins shedding neoplastic cells into circulation at an early stage (18). The amount of free plasma DNA in circulation is 5- to 10-fold higher in patients with solid tumours than that in normal patients (19). In this study, we used PNA clamp real-time PCR to detect KRAS mutations in blood samples of CRC patients. This assay is useful for detecting mutations in codons 12 and 13 of the KRAS gene and is based on high-fidelity DNA polymerase. To detect a minimal amount of mutant DNA in clinical samples, PNA oligomers have been developed. PNAs are non-extendable oligonucleotides in which the ribose-phosphate backbone is replaced by peptide bondlinked 2-aminoethyl glycine units. In PNA clamp PCR, PNA oligomers suppress the amplification of the complementary sequence confined by a pair of DNA oligonucleotide primers since PNA is not a substrate for DNA polymerase (20). We believe that our method of detecting KRAS mutations in blood samples of metastatic CRC patients may be a good clinical tool for predicting the effectiveness of EGFR-targeted monoclonal antibodies.

Lindforss et al (19) reported that plasma KRAS mutants could be detected preoperatively and early postoperatively in all stages of colorectal neoplasia. They found that 8 of 9 patients, all of whom were radically treated, still displayed tumour-derived KRAS mutants in their plasma on day 3 after surgical resection. The result of our experiment was contrary 
to that of Lindforss et al (19). In our study, all 5 patients with preoperative plasma KRAS mutants did not show KRAS point mutations 6 months postoperatively.

In conclusion, our method was able to detect $K R A S$ point mutations in the peripheral blood of CRC patients, which contains extremely small amounts of mutant cells. This method is helpful for identifying metastatic CRC patients in whom their metastases will respond to EGFR-targeted monoclonal antibodies. Furthermore, it is hopeful that this method will reveal micrometastases in CRC patients as tumour markers. It is likely that this method can substitute for previous methods of detecting KRAS point mutations in almost all CRC patients.

\section{Acknowledgements}

The authors thank Takafumi Fukui, Yosuke Furui and Hideaki Maruse of the Biomedical Business Department, Falco Biosystems Ltd. for performing the KRAS mutation detection assays.

\section{References}

1. Parkin DM, Bray F, Ferlay J and Pisani P: Global cancer statistics, 2002. CA Cancer J Clin 55: 74-108, 2005.

2. Wolpin BM and Mayer RJ: Systemic treatment of colorectal cancer. Gastroenterology 134: 1296-1310, 2008.

3. Chung KY, Shia J, Kemeny NE, Shah M, Schwartz GK, Tse A, Hamilton A, Pan D, Schrag D, Schwartz L, et al: Cetuximab shows activity in colorectal cancer patients with tumors that do not express the epidermal growth factor receptor by immunohistochemistry. J Clin Oncol 20: 1803-1810, 2005.

4. Bokemeyer C, Bondarenko I, Hartmann JT, de Braud F, Schuch G, Zubel A, Celik I, Schlichting M and Koralewski P: Efficacy according to biomarker status of cetuximab plus FOLFOX-4 as first-line treatment for metastatic colorectal cancer: the OPUS study. Ann Oncol 22: 1535-1546, 2011.

5. Van Cutsem E, Köhne CH, Hitre E, Zaluski J, Chang Chien CR, Makhson A, D'Haens G, Pintér T, Lim R, Bodoky G, et al: Cetuximab and chemotherapy as initial treatment for metastatic colorectal cancer. N Engl J Med 360: 1408-1417, 2009.

6. Walther A, Johnstone E, Swanton C, Midgley R, Tomlinson I and Kerr D: Genetic prognostic and predictive markers in colorectal cancer. Nat Rev Cancer 9: 489-499, 2009.

7. Bardelli A and Siena SJ: Molecular mechanisms of resistance to cetuximab and panitumumab in colorectal cancer. Clin Oncol 28: 1254-1261, 2010.
8. Winder T and Lenz HJ: Vascular endothelial growth factor and epidermal growth factor signaling pathways as therapeutic targets for colorectal cancer. Gastroenterology 138: 2163-2176, 2010.

9. Chua W, Goldstein D, Lee CK, Dhillon H, Michael M, Mitchell P, Clarke SJ and Iacopetta B: Molecular markers of response and toxicity to FOLFOX chemotherapy in metastatic colorectal cancer. Br J Cancer 101: 998-1004, 2009.

10. Gilje B, Heikkilä R, Oltedal S, Tjensvoll K and Nordgard O: High-fidelity DNA polymerase enhances the sensitivity of a peptide nucleic acid clamp PCR assay for K-ras mutations. Mol Diagn 10: 325-331, 2008.

11. Forbes S, Clements J, Dawson E, Bamford S, Webb T, Dogan A, Flanagan A, Teague J, Wooster R, Futreal PA and Stratton MR: COSMIC 2005. Br J Cancer 94: 318-322, 2006.

12. Poehlmann A, Kuester D, Meyer F, Lippert H, Roessner A and Schneider-Stock R: K-ras mutation detection in colorectal cancer using the Pyrosequencing technique. Pathol Res Pract 203: 489-497, 2007.

13. Raponi M, Winkler $\mathrm{H}$ and Dracopoli NC: KRAS mutations predict response to EGFR inhibitors. Curr Opin Pharmacol 8: 413-418, 2008

14. Saif MW and Shah M: K-ras mutations in colorectal cancer: a practice changing discovery. Clin Adv Hematol Oncol 7: 45-53, 2009.

15. Klein CA: Parallel progression of primary tumours and metastases. Nat Rev Cancer 9: 302-312, 2009.

16. Knijn N, Mekenkamp LJ, Klomp M, Vink-Börger ME, Tol J, Teerenstra S, Meijer JW, Tebar M, Riemersma S, van Krieken JH, et al: KRAS mutation analysis: a comparison between primary tumours and matched liver metastases in 305 colorectal cancer patients. Br J Cancer 104: 1020-1026, 2011.

17. Vogelstein B, Fearon ER, Hamilton SR, Kern SE, Preisinger AC, Leppert M, Nakamura Y, White R, Smits AM and Bos JL: Genetic alterations during colorectal-tumor development. N Engl J Med 319: 525-532, 1988

18. Wang JY, Yeh CS, Chen YF, Wu CH, Hsieh JS, Huang TJ, Huang SY and Lin SR: Development and evaluation of a colorimetric membrane-array method for the detection of circulating tumor cells in the peripheral blood of Taiwanese patients with colorectal cancer. Int J Mol Med 17: 737-747, 2006.

19. Lindforss U, Zetterquist $\mathrm{H}$, Papadogiannakis $\mathrm{N}$ and Olivecrona $\mathrm{H}$ : Persistence of K-ras mutations in plasma after colorectal tumor resection. Anticancer Res 25: 657-661, 2005.

20. Beau-Faller M, Legrain M, Voegeli AC, Guérin E, Lavaux T, Ruppert AM, Neuville A, Massard G, Wihlm JM, Quoix E, et al: Detection of K-ras mutations in tumor samples of patients with non-small cell lung cancer using PNA-mediated PCR clamping. Br J Cancer 100: 985-992, 2009. 